\title{
Mode-Selective Chemistry through Polaritonic Vibrational Strong Coupling
}

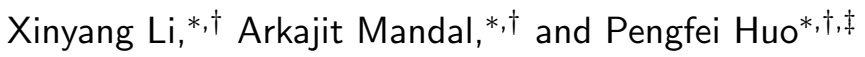 \\ $\dagger$ Department of Chemistry, University of Rochester, Rochester, NY, 14627 \\ $\ddagger$ Institute of Optics, University of Rochester, Rochester, New York, 14627, USA
}

E-mail: xinyang.li@rochester.edu; amandal4@ur.rochester.edu; pengfei.huo@rochester.edu

\section{Abstract}

Recent experiments have demonstrated remarkable mode-selective reactivities by coupling molecular vibrations with vacuum fluctuations inside an optical cavity. The fundamental mechanism behind such effects, on the other hand, remains elusive. In this work, we theoretically demonstrate the basic principle of how cavity photon frequency can be tuned to achieve mode-selective reactivities. We find that the non-Markovian nature of the radiation mode leads to a cavity frequency-dependent dynamical caging effect of a reaction coordinate, resulting in a suppression of the rate constant. In the presence of multiple competitive reactions, it is possible to preferentially cage a reaction coordinate when the barrier frequencies for competing reaction paths are different. Our theoretical results illustrate the cavityinduced mode-selective chemistry through polaritonic vibrational-strong couplings, revealing the fundamental mechanism for changing chemical selectivities through cavity quantum electrodynamics.

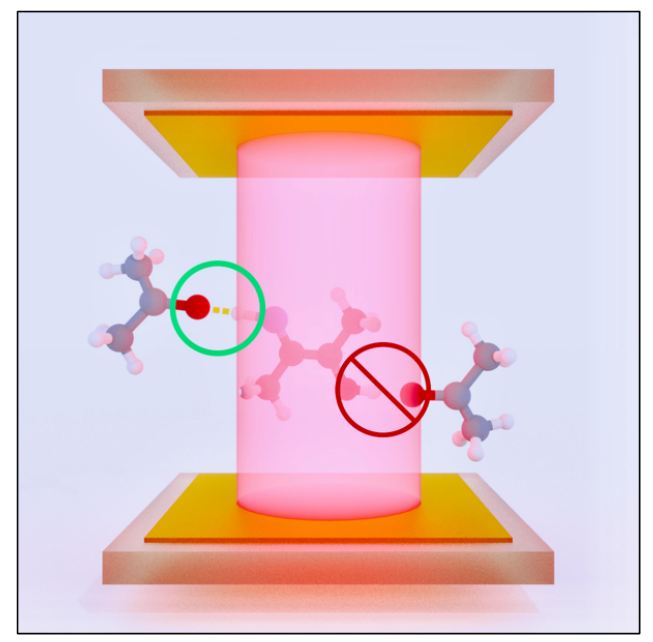

Introduction. Polariton Chemistry is an emerging field ${ }^{1-5}$ that provides opportunities for new chemical reactivities or selectivities by coupling molecular systems to quantized radiation fields inside an optical cavity. By hybridizing vibrational excitations of the molecule with the photonic excitation of the radiation inside the cavity, new light-matter entangled states, so-called the polariton states are generated. Recently, it has been demonstrated that it is possible to suppress ${ }^{6-10}$ or enhance ${ }^{11,12}$ ground state chemical reactivities by placing an ensemble of molecules in an optical micro-cavity through the resonant coupling between the cavity and vibrational degrees of freedom (DOF) of the molecules. This so-called vibrational strong coupling (VSC) regime ${ }^{5}$ operates in the $a b$ sence of any light source, ${ }^{7,8}$ and was hypothesized to utilize the hybridization of a vibrational transition of a molecule and the zero-point energy fluctuations of a cavity mode. ${ }^{7,8}$

In a recent ground-breaking experiment, Ebbesen and co-workers ${ }^{7}$ have demonstrated that the cavity can selectively slow down a particular reaction among competing reaction pathways, and revert the reactive selectivities. This new strategy of VSC, if feasible, will allow one to bypass some intrinsic difficulties (such as intramolecular vibrational energy transfer) encountered in the modeselective chemistry that uses IR excitation to tune chemical reactivities, offering a paradigm-shift of synthetic chemistry through cavity enabled bondselective chemical transformations. ${ }^{7,8}$

Unfortunately, a clear theoretical understanding of such remarkable VSC ground state reactivities remains missing, including the explanation of resonant effect where the suppression of the rate is achieved with a particular cavity photon frequency as well as collective coupling effects. In addition, 
the reported branching ratios tilt toward the same product regardless of whether the $\mathrm{Si}-\mathrm{C}$, Si-O, or $\mathrm{Si}-\mathrm{CH}_{3}$ mode is resonant with the cavity. ${ }^{7}$ A reasonable theoretical explanation for this observed kinetics modification remains elusive.

In response to this theoretical challenge, we have recently conjectured that the non-Markovian nature of a cavity radiation mode could lead to a significant suppression of the chemical reaction rate constant at a particular photon frequency that is related to the reaction barrier frequency. ${ }^{13}$ At a particular frequency, the cavity radiation mode induces the dynamical-caging effect, ${ }^{13-15}$ such that the molecular reaction coordinate becomes trapped in a narrow "photonic solvent cage" near to the top of the barrier region, leading to a suppression of the chemical kinetics. Such effects are dynamical and are not captured within a simple transition state theory. ${ }^{16}$

In this work, we use the dynamical caging conjecture of VSC polariton chemistry ${ }^{13}$ to explore the possibility of cavity enabled mode selective chemistry. ${ }^{7}$ We consider a model molecular system that has two competing reaction pathways, with an almost identical potential energy barrier height (with the difference less than $0.2 \mathrm{kcal} / \mathrm{mol}$ ), but different imaginary barrier frequencies. We demonstrate that by tuning the cavity frequency, one can selectively cage one reaction channel over the other, hence changing the ratio of the rate constants of two reactions as well as the preference of competing reactions.

Theoretical Model. For a molecule system $\hat{H}_{\mathrm{M}}$ coupled to a quantized photon mode inside an optical cavity, the Pauli-Fierz (PF) QED Hamiltonian ${ }^{17-20}$ is expressed as follows

$$
\hat{H}=\hat{H}_{\mathrm{m}}+\frac{\hat{p}_{\mathrm{c}}^{2}}{2}+\frac{1}{2} \omega_{\mathrm{c}}^{2}\left(\hat{q}_{\mathrm{c}}+\sqrt{\frac{2}{\hbar \omega_{\mathrm{c}}^{3}}} \chi \cdot \mu(\mathbf{R})\right)^{2},
$$

where $\hat{H}_{\mathrm{m}}$ is the molecular Hamiltonian and $\mu(\mathbf{R})$ is the total dipole operator associated with the molecule. Further, $\hat{q}_{\mathrm{c}}=\sqrt{\hbar / 2 \omega_{\mathrm{c}}}\left(\hat{a}^{\dagger}+\hat{a}\right)$ and $\hat{p}_{\mathrm{c}}=i \sqrt{\hbar \omega_{\mathrm{c}} / 2}\left(\hat{a}^{\dagger}-\hat{a}\right)$ are the photon mode coordinate and momentum operator, respectively, where $\hat{a}^{\dagger}$ and $\hat{a}$ are the photon mode creation and annihilation operators. Under the dipole gauge, the matter interacts with the quantized radiation mode of the cavity by displacing the photonic coordinate with the amount of $\sqrt{\frac{2}{\hbar \omega_{\mathrm{c}}^{3}}} \chi \cdot \mu(\mathbf{R})$, where $\chi$ characterizes the coupling strength between the molecule and the cavity. Note that the correct QED description in Eq. 3 includes the dipole self-energy (DSE) $(\chi \cdot \mu(\mathbf{R}))^{2} / \hbar \omega_{\mathrm{c}}$. A detailed derivation of this Hamiltonian is provided in the Supporting Information.

We further define a reduced coupling strength

$$
\tilde{\eta}=\frac{\chi}{\sqrt{M} \omega_{\mathrm{c}}}
$$

which is used to characterize the coupling strength in the system regardless $\omega_{\mathrm{c}}$. At the resonant condition to observe Rabi splitting in absorption spectra, which is $\omega_{\mathrm{c}}=\omega_{0}$, the photon-vibration interaction couples photon-dressed vibronic-Fock states $\left|\nu_{0}, 1\right\rangle$ (photonic excitation) and $\left|\nu_{1}, 0\right\rangle$ (vibrational excitation), inducing a Rabi splitting in the absorption spectra as $\hbar \Omega_{R}=2 \sqrt{\frac{\hbar}{2 M \omega_{0}}} \chi \cdot \mu_{0}^{\prime} \equiv 2 \frac{\mu_{0}^{\prime}}{\sqrt{2 \hbar \omega_{0}}} \cdot \hbar \omega_{c} \cdot \tilde{\eta}$. Here, $\mu_{0}^{\prime}=d \mu(R) /\left.d R\right|_{R=R_{0}}$ is the slope of the dipole around the minima $R_{0}$.

In this study, we consider a molecule that can undergo two competing reaction pathways, depicted in Fig. 1a. We model these two competing reactions with two independent Shin-Metiu models. ${ }^{21}$ We assume the directions of the two reaction are completely parallel, such that both can be aligned with the cavity polarization direction. This is similar to the model molecular systems investigated by Ebbesen and co-workers. ${ }^{7}$ The molecular Hamiltonian is

$$
\hat{H}_{\mathrm{m}}=\sum_{i=1}^{2} \frac{\hat{P}_{i}^{2}}{2 M_{i}}+E\left(R_{1}, R_{2}\right)+\hat{H}_{\mathrm{vib}}(\mathbf{R}, \mathbf{x}),
$$

where $E\left(R_{1}, R_{2}\right)=E_{1}\left(R_{1}\right)+E_{2}\left(R_{2}\right)$ is the ground electronic state potential energy surface for two uncoupled Shin-Metiu model, which are depicted in Fig. 1b. The total dipole is modeled as $\mu(\mathbf{R})=$ $\mu_{1}\left(R_{1}\right)+\mu_{2}\left(R_{2}\right)$, of the $i_{\text {th }}$ reaction coordinate depicted in the top panels of Fig. 1b, $\mu_{i}\left(R_{i}\right)$ is the ground state permanent dipole moment of the i-th reaction coordinate depicted in the bottom panels of Fig. 1b. In addition, $\hat{H}_{\text {vib }}$ (see details in the Supporting Information) is the vibrational systembath Hamiltonian that describes the interactions between reaction coordinates $R_{1}$ and $R_{2}$ and other vibrational phonon modes $\mathbf{x}$ in the molecule.

The VSC polariton chemical kinetics can be viewed as a barrier crossing process on the cavity 
Born-Oppenheimer surface (CBO), ${ }^{17,22,23}$

$$
\begin{aligned}
& V_{\mathrm{CBO}}\left(\mathbf{R}, q_{\mathrm{c}}\right) \\
& =\sum_{i=1}^{2} E_{i}\left(R_{i}\right)+\frac{1}{2} \omega_{\mathrm{c}}^{2}\left(\hat{q}_{\mathrm{c}}+\sqrt{\frac{2}{\hbar \omega_{\mathrm{c}}^{3}}} \chi \cdot \sum_{i=1}^{2} \mu_{i}\left(R_{i}\right)\right)^{2} .
\end{aligned}
$$

In the VSC regime, the cavity mode has a similar range of frequency as the molecular vibrations, meaning that $q_{\mathrm{c}}$ evolve at a similar time scale as R. Following previous works, ${ }^{23-25}$ here we treat all nuclear and photonic DOF classically.

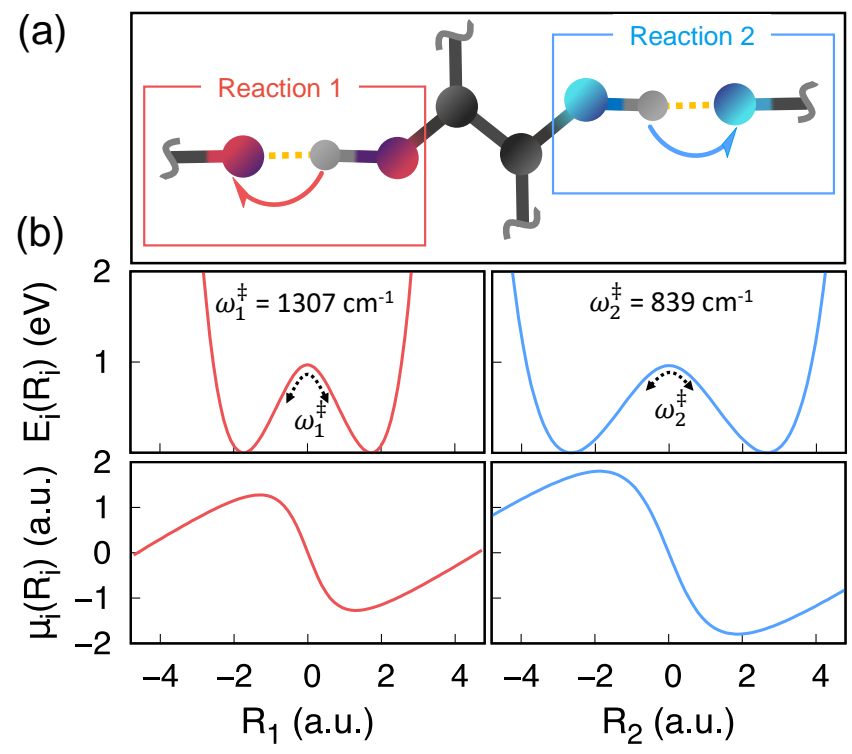

Figure 1: (a) A model molecular system with two competing reactions pathways, labeled as Reaction 1 (red) and Reaction 2 (blue), respectively. (b) The potential energy surface of the electronic ground state Shin-Metiu models used to simulate the PCET reactions. The ground state potential energy surfaces (PES) $E(R)$ are shown in the top panels, and the permanent dipole moment $\mu(R)$ are shown in the bottom panels. All plots are in the same color coding as panel (a). The reaction barrier of Reaction $2\left(\omega_{2}^{\ddagger}=839 \mathrm{~cm}^{-1}\right)$ is much broader than that of Reaction $1\left(\omega_{1}^{\ddagger}=1307 \mathrm{~cm}^{-1}\right)$.

Reaction Rate Constant. Formally, the rate constant can be rigorously expressed in terms of the TST rate $k_{\mathrm{TST}}$ and the transmission coefficient $\kappa$ as follows

$$
k=\lim _{t \rightarrow t_{\mathrm{p}}} \kappa(t) \cdot k_{\mathrm{TST}},
$$

where $t_{\mathrm{p}}$ refers to the plateau time of the flux-side correlation function, and $\kappa(t)$ is the transmission coefficient that captures the dynamical recrossing effects, which measures the ratio between the actual reaction rate and the TST rate. We will also refer to the plateau value of the flux-side correlation function as $\kappa\left(t_{\mathrm{p}}\right)$. The TST rate is expressed as

$$
k_{\mathrm{TST}}=\frac{\omega^{0}}{2 \pi} e^{-\beta E_{\mathrm{b}}},
$$

where $E_{\mathrm{b}}=E\left(R^{\ddagger}\right)-E\left(R^{0}\right)$ is the potential energy barrier height measured from the bottom of the well $R^{0}$ to the top of the barrier $R^{\ddagger}$, and $\omega^{0}$ is the vibrational frequency of the reactant at $R=R^{0}$, and $\beta=\left(k_{\mathrm{B}} T\right)^{-1}$. When the DSE is explicitly considered, $E_{\mathrm{b}}$ remains invariant as changing the light-matter coupling strength or the photon frequency, explaining why one can not observe any effects from a simple TST analysis. ${ }^{24-26}$

Since $k_{\text {TST }}$ does not change under the VSC condition, we have conjectured ${ }^{13}$ that VSC chemical reactivities is purely originated from the transmission coefficient $\kappa$. We can compute $\kappa$ through calculating the flux-side correlation function numerically, ${ }^{27-29}$ which is defined as

$$
\kappa(t)=\frac{\left\langle\mathcal{F}(0) \cdot h\left[R(t)-R^{\ddagger}\right]\right\rangle}{\left\langle\mathcal{F}(0) \cdot h\left[\dot{R}^{\ddagger}(0)\right]\right\rangle},
$$

where $h\left[R-R^{\ddagger}\right]$ is the Heaviside function of the reaction coordinate $R$, with the dividing surface $R_{\ddagger}$ that separate the reactant and the product regions (for the model system studied here, $R^{\ddagger}=0$ ), the flux function $\mathcal{F}(t)=\dot{h}(t)=\delta\left[R(t)-R^{\ddagger}\right] \cdot \dot{R}(t)$ measures the reactive flux across the dividing surface (with $\delta(R)$ as the Dirac delta function), and $\langle\ldots\rangle$ represents the canonical ensemble average (subject to the constraint on the dividing surface which is enforced by $\delta\left[R(t)-R_{\ddagger}\right]$ inside $\left.\mathcal{F}(t)\right)$. Further, $\dot{R}^{\ddagger}(0)$ represents the initial velocity of the nuclei on the dividing surface. The above flux-side formalism of the reaction rate can be derived from Onsager's regression hypothesis, with derivations presented in standard text books (e.g., Ref. 29). The numerical simulation details of $\kappa$ is provided in the Supporting Information.

Further, the total rate constant of the model system can be estimated from the Grote-Hynes Rate Theory. ${ }^{14,15,30}$ The GH rate constant is given as $^{30-33}$

$$
k_{\mathrm{GH}}=\frac{1}{2 \pi} \frac{\prod_{\nu=1}^{N} \Omega_{\nu}^{0}}{\prod_{\nu=2}^{N} \Omega_{\nu}^{\ddagger}} e^{-\beta E_{b}},
$$

where $\left\{\Omega_{\nu}^{0}\right\}$ are normal mode frequencies of the Hamiltonian in the reactant well, and for $\nu \geq 2$, $\left\{\Omega_{\nu}^{\ddagger}\right\}$ is the stable normal mode frequency at the 
barrier, such that $\Omega_{\nu}^{\ddagger 2}>0$. For $\nu=1, \Omega_{\nu}^{\ddagger 2}<0$ is the imaginary frequency of the transition state. Treating $\mathbf{R}$ and $q_{\mathrm{c}}$ as the "coordinates" of the hybrid system, we directly diagonalize the Hessian Matrix of the model Hamiltonian (Eq. 4) to compute these frequencies to obtain the $\mathrm{GH}$ rate. The details of these calculations are provided in the Supporting Information.
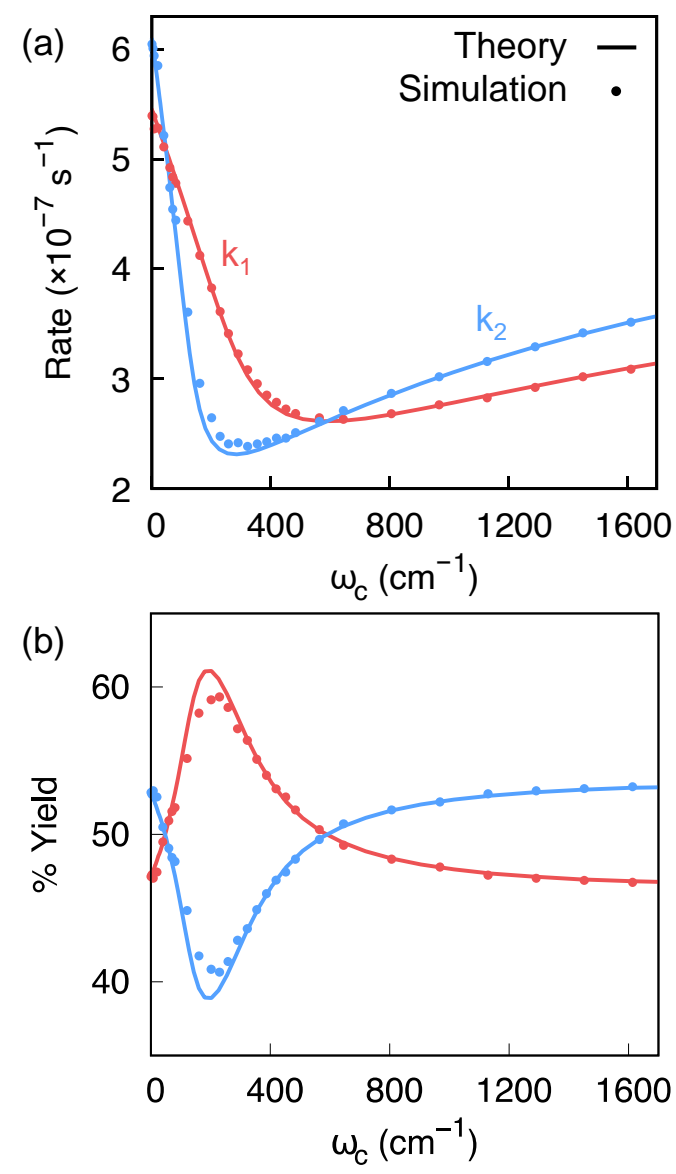

Figure 2: (a) The chemical reaction rate constants of the two competing pathways 1 (red) and 2 (blue) at various cavity frequency $\omega_{\mathrm{c}}$ obtained from numerical simulations (dots) based on Eq. 5 as well as the analytical rate expression (solid curve) in Eq. 8. (b) The yield (\%) $\phi_{\mathrm{i}}$ (Eq. 9) of reaction $i$ as a function of $\omega_{\mathrm{c}}$. The preference of the product is reversed in the region of $\omega_{\mathrm{c}} \in\left[49 \mathrm{~cm}^{-1}, 565 \mathrm{~cm}^{-1}\right]$.

The yield of the reaction $i$ is estimated as ${ }^{7}$

$$
\phi_{i}=\frac{k_{i}}{k_{1}+k_{2}},
$$

for two competing pathways $i \in\{1,2\}$, where the reaction rate constants $k_{\mathrm{i}}=\kappa_{\mathrm{i}}\left(t_{\mathrm{p}}\right) \cdot k_{\mathrm{TST}}(i), \kappa\left(t_{\mathrm{p}}\right)$ can be computed based on Eq. 6 and Eq. 7, or using the GH analytical rate theory in Eq. 8.

Results and Discussions. Fig. 2a presents the reaction rate constants $k_{\mathrm{i}}$ of two competing pathways $i \in\{1,2\}$ as a function of cavity frequency $\omega_{\mathrm{c}}$ under the normalized coupling strength (see Eq. 2) $\tilde{\eta}=0.047$ a.u.. One can clearly see that the two reaction rate constants $k_{1}$ and $k_{2}$ are suppressed with the present of the cavity, agreeing with recently observed rate constant suppression of the VSC chemistry. We emphasize that this suppression is completely originated from the reduction of the transmission coefficient $\kappa$ (Eq. 7), not from the TST rate constant (Eq. 6) as the classical barrier height does not change with the presence of cavity. Interestingly, the maximum suppression of $k_{\mathrm{i}}$ occur at two different cavity frequency. This cavity frequency-dependent reduction of rate constant has been explained from our earlier work: ${ }^{13}$ when the cavity frequency reaches to a frequency related to the top of the barrier frequency $\omega_{\mathrm{b}}$, the dynamical cadging effect reaches to its largest magnitude. More specifically, for a simplified model with one only reaction coordinate and without the phonon bath $\hat{H}_{\text {vib }}$ (in Eq. 3), the maximum suppression frequency is

$$
\omega_{\mathrm{s}}=-\frac{\hbar}{2} \tilde{\eta}^{2} \mu_{\ddagger}^{\prime 2}+\frac{1}{2} \sqrt{\hbar^{2} \tilde{\eta}^{4} \mu_{\ddagger}^{\prime 4}+4 \omega_{\mathrm{b}}^{2}},
$$

where $\tilde{\eta}=\frac{\chi}{\sqrt{M} \hbar \omega_{\mathrm{c}}}$ and $\mu_{\ddagger}^{\prime}=\left.\frac{\partial \mu(R)}{\partial R}\right|_{R^{\ddagger}}$. The presence of $\hat{H}_{\text {vib }}$ does not significantly change this frequency. The detailed derivation and discussions are provided in the Supporting Information. When $\tilde{\eta}$ is small such that $\tilde{\eta}^{4} \mu_{\ddagger}^{\prime 4} \ll 4 \omega_{\mathrm{b}}^{2}$, the the maximum suppression frequency is close to the barrier frequency, such that $\omega_{\mathrm{s}} \approx \omega_{\mathrm{b}}$. As the coupling strength $\tilde{\eta}$ increases, the minimum will be shifted to the low-frequency region, as the scenario demonstrated in Fig. 2a. Nevertheless, the difference in $\omega_{\mathrm{b}}$ associated within two competing reactions manifest into the difference of the maximum suppression frequencies associated with two reactions.

Fig. $2 \mathrm{~b}$ presents the yield of the product ${ }^{7} \phi_{i}$ (defined in Eq. 9) for reaction $i$ as a function of $\omega_{\mathrm{c}}$. As one can see, the changing preference of the yield is rooted in the frequency-dependent suppression of the rate constant $k_{i}$ shown in Fig. $2 \mathrm{~b}$. In particular, the reverted preference occurs at a range of different cavity frequencies, in the region of $\omega_{\mathrm{c}} \in\left[49 \mathrm{~cm}^{-1}, 565 \mathrm{~cm}^{-1}\right]$, and the maximum reverted preference occurs at $\omega_{\mathrm{c}}=201.6 \mathrm{~cm}^{-1}$, where the ratio $k_{2} / k_{1}$ has been changed in the most significantly by coupling to the cavity (because $\phi_{1}=1 /\left(1+k_{2} / k_{1}\right)$. Even though that our model 

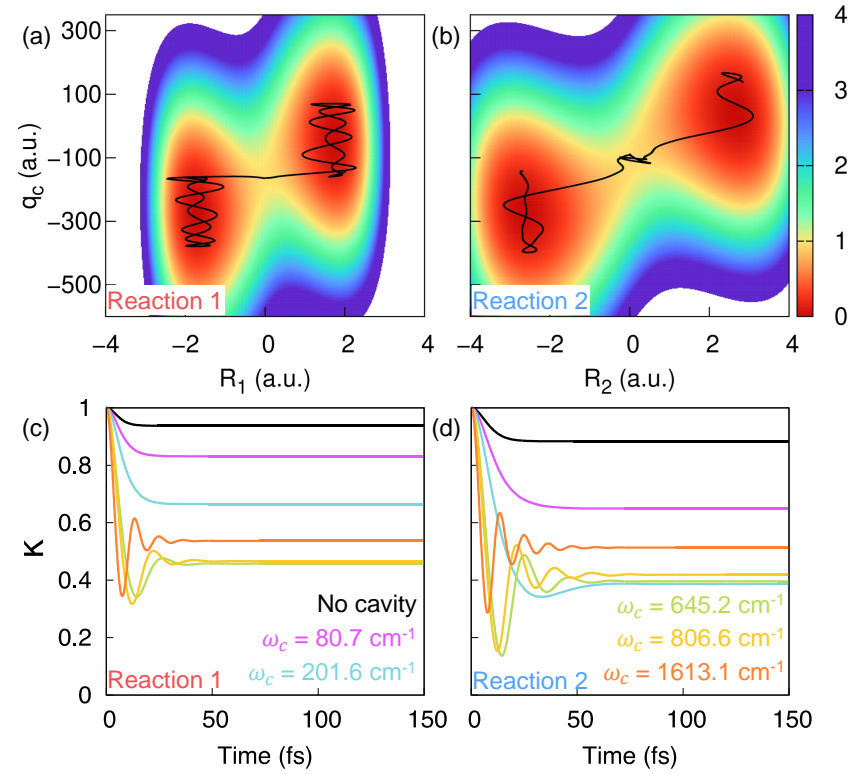

Figure 3: (a) The Cavity Born-Oppenheimer (CBO) surface and a representative trajectory for Reaction 1 at $\omega_{\mathrm{c}}=201.6 \mathrm{~cm}^{-1}$. The reaction coordinate $R_{2}$ is fixed at its equilibrium position for plotting the surface. (b) CBO surface for Reaction 2 at $\omega_{\mathrm{c}}=201.6 \mathrm{~cm}^{-1}$, which exhibit the "dynamical caging" effect from the photon field (recrossing in the barrier region). The transmission coefficient $\kappa(t)$ at various cavity frequency $\omega_{\mathrm{c}}$ are presented for (c) reaction 1 and (d) reaction 2.

system is different than the molecular system explored by Ebbesen and co-workers, ${ }^{7}$ our results show some interesting basic features of that experiment. In particular, we find that using a highfrequency off-resonant cavity $\left(\omega_{\mathrm{c}}\right.$ is larger than all vibrational frequencies, such as $\omega_{\mathrm{c}}>1600 \mathrm{~cm}^{-1}$ in the current model), the selectivity is the same as the original selectivity without the cavity (effectively $\left.\omega_{\mathrm{c}}=0\right)$. Further, we find that the reverted preference occurs during a range of cavity frequencies, even though the maximum reduction of the rate constants for two competing reactions occur at two specific cavity frequencies. Our theoretical results provide a new perspective to understand the recent VSC enhanced selectivities of competing reactions, such as the results presented in Ref. 7 (in particular, Fig. 3b in that work) which demonstrate a similar trend of change of preference at three different cavity frequencies. To further understand the reverted selectivities of reactions, we further explore representative reactive trajectories on the cavity BO surfaces for both reactions.

Fig. 3a presents the CBO surface along the photonic coordinate $q_{\mathrm{c}}$ as well as reactive coordinate $R_{1}$, whereas $R_{2}$ is fixed at the equilibrium posi- tion $R_{2}^{0}$. The molecule is coupled to a cavity with $\omega_{\mathrm{c}}=201.6 \mathrm{~cm}^{-1}$ through the coupling strength $\tilde{\eta}=0.047$ a.u. A representative trajectory for the molecule undergoes reaction 1 (black solid line) is also presented on top of the CBO surface. Because $\omega_{\mathrm{c}}$ is detuned from the maximum suppression frequency $\omega_{\mathrm{s}}$ (Eq. 10), the friction from the photonic coordinate $q_{\mathrm{c}}$ does not severely impede the transitions of $R_{1}$. The majority of the trajectories of reaction 1 directly pass through the transition state region so that the transmission coefficient $\kappa$ is not significantly reduced compared to the value in the no-coupling case.

Fig. $3 \mathrm{~b}$ presents the CBO surface along $q_{\mathrm{c}}$ and $R_{2}$, as well as a representative reactive trajectory along for reaction 2 under the same cavity frequency and coupling strength as used in Fig. 3a. For reaction 2 , the cavity frequency equals to the maximum suppression frequency $\omega_{\mathrm{c}}=\omega_{\mathrm{s}}$, and the reaction coordinate $R_{2}$ becomes trapped in a narrow "solvent cage" on the barrier top, hence significantly slows down $\kappa_{2}$ through multiple recrossing dynamics (as shown in Fig. 3b). Together with Fig. 3a, we find that the fundamental mechanism of the cavity enhanced selectivities is originated from a selective "solvent caging effect" (i.e., enhancement of the recrossing dynamics) among two competing reactions, when the cavity frequency is selectively tuned to match the maximum suppression frequency $\omega_{\mathrm{s}}$ of one reaction, but not the other.

Fig. 3c-d presents the time-dependent transmission coefficient $\kappa(t)$ (see Eq. 7) for (c) reaction 1 and $(d)$ reaction 2 at five different cavity frequencies. Note that as $\omega_{\mathrm{c}}$ increases, $\kappa(t)$ for both reactions becomes more oscillatory. The long time plateau values of $\kappa(t)$ keep decreasing when increasing the cavity frequency, after reaching to a minimum (when $\omega_{\mathrm{c}}$ equals to the $\omega_{\mathrm{s}}$ for a given reaction), $\kappa(t)$ starts increase again. This can be clearly see from the rate constant presented in Fig. 2. This cavity-frequency dependent suppression of rate constant has been extensively discussed in our previous work: ${ }^{13}$ when $\omega_{\mathrm{c}}$ close to the $\omega_{\mathrm{s}}$, the dynamical caging effects reaches to its maximum magnitude, and when $\omega_{\mathrm{c}}$ deviate from $\omega_{\mathrm{s}}$ the reactive channel on CBO surface is either very broad or very narrow, such that the reactive trajectory is not severely caged compared to the condition of $\omega_{\mathrm{c}}=\omega_{\mathrm{s}}$.

Fig. 4a presents the reaction rate constants $k_{i}$ of two competing pathways at a fixed photon mode frequency $\omega_{\mathrm{c}}=201.6 \mathrm{~cm}^{-1}$ while changing the 

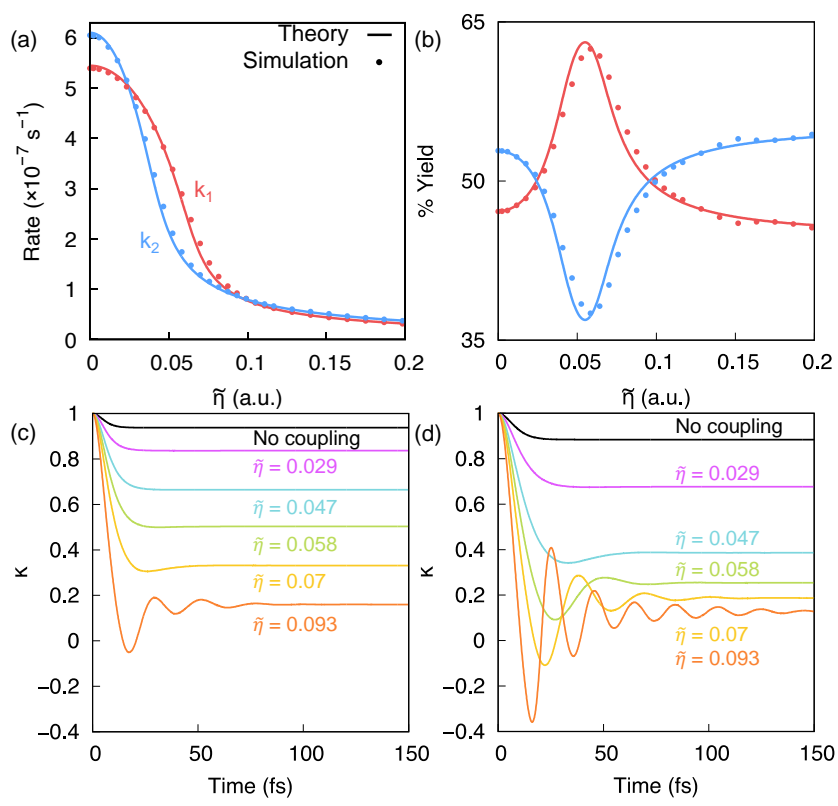

Figure 4: Selectivity with respect to changing coupling strength. (a) Reaction rate constants of reaction 1 (red) and reaction 2 (blue) versus the reduced coupling strength $\tilde{\eta}=\chi /\left(\sqrt{M} \omega_{\mathrm{c}}\right)$. (b) The percentage yield. (c) \& (d) The time-dependent transmission coefficient $\kappa(t)$ at different coupling strength for reaction 1 (left) and 2 (right).

normalized coupling strength $\tilde{\eta}$ (Eq. 2). The rate constants of both pathways decrease monotonically as $\tilde{\eta}$ increases, due to the transition from the non-equilibrium solvation regime to the dynamical caging regime as discussed in our previous work. ${ }^{13}$

Fig. $4 \mathrm{~b}$ presents the yield $\phi_{i}$ associated with both pathways, with an interesting switch over of the reaction preference also occur at an intermediate coupling strength. To the best of our knowledge, this effect has not been experimentally explored. This theoretical prediction suggests that tuning the light-matter coupling strength can also reverse the preference of the reaction. For our case, the maximum selection is achieved at $\tilde{\eta}=0.058$ a.u. As the coupling strength enters into the ultra strong coupling regime, the yield for both products goes back to the value of the cavity free scenario, as both reactions are severely caged. ${ }^{13}$

Fig. 4c-d presents the transmission coefficient $\kappa(t)$ at various $\tilde{\eta}$. Similar to the $\kappa(t)$ plots in Fig. 3c-d, here, $\kappa(t)$ becomes more oscillatory as the coupling strength $\tilde{\eta}$ increases. However, there is no turnover in the plateau value $\kappa\left(t_{\mathrm{p}}\right)$ in this case, in contrast to the scenario presented in Fig. 3c-d. On the other hand, the Rabi splitting (from the IR spectrum) of the current work is within the range of the recent VSC experiments. ${ }^{7,8}$ This is because in these VSC experiments, the collective coupling strength is scaled up by $\sqrt{N}$. In this study, we have also explicitly assumed that the dipole moment is always aligned with the cavity polarization direction.

Conclusions. In this work, we demonstrate the possibility of cavity enabled mode-selective chemistry originated from the "dynamical caging" effect from the photon field, causing a maximum suppression on a reaction rate constant at a frequency related to the original imaginary barrier frequency of the reaction pathway. We show that the ratio of the two rate constants, hence, the selectivity of two competing reaction pathways can be reverted when the two pathways have different barrier frequencies. This is because that under a specific cavity frequency, one reaction is not as caged as the other one due to difference for their top of the barrier frequencies. Under the strong light-matter coupling strengths, the preferred products can be reverted at a range of cavity frequencies. The reactive preference when the cavity frequency is much higher than all vibrational frequencies (off-resonant condition) remains the same as the original preference outside the cavity. These findings reproduces the basic features observed in the recent

In addition, we also predict that at a fixed cavity frequency, the selectivity can also be tuned by changing the coupling strength of the light-matter interaction. Similar to the cavity frequency tuning, the maximum selectivity can be achieved at an intermediate coupling strength. Further increasing the coupling strength will result in the same selectivity as the no-cavity case. To the best of our knowledge, this effect has not been observed experimentally. We encourage future experimental works to explore this theoretical prediction.

Admittedly, the cavity frequencies where the maximum suppression occurs show a strong red shift with respect to the original barrier frequencies of both pathways, due to a large coupling strength we chose. As we explained in our previous work, ${ }^{13}$ the experiments are in the collective coupling regime, ${ }^{7}$ whereas our work is limited to the case where a few molecule is strongly coupled to a single radiation mode. Future theoretical work is needed to be done to investigate the collective effects in VSC.. ${ }^{24,25}$ On the other hand, the experimental set up of the single molecule-polasmonic cavity ${ }^{34}$ should allow the direct verification of the theoretical conjectures presented in this work. 
To summarize, our work provides a plausible theoretical explanation on the mode-selective chemistry through polaritonic vibrational strong coupling. The rate constants of two competing reactions are unevenly suppressed from the dynamical caging effect originated from the molecule-cavity interactions. Further investigation will focus on understanding the collective VSC reactivities by coupling many molecules with the cavity. ${ }^{24,25}$

\section{Acknowledgments}

This work was supported by the National Science Foundation CAREER Award under Grant No. CHE-1845747, as well as by a Cottrell Scholar award (a program by of Research Corporation for Science Advancement). Computing resources were provided by the Center for Integrated Research Computing (CIRC) at the University of Rochester.

\section{References}

(1) Ebbesen, T. W. Hybrid Light-Matter States in a Molecular and Material Science Perspective. Acc. Chem. Res. 2016, 49, 2403-2412.

(2) Ribeiro, R. F.; Martínez-Martínez, L. A.; Du, M.; Campos-Gonzalez-Angulo, J.; Yuen-Zhou, J. Polariton Chemistry: Controlling Molecular Dynamics With Optical Cavities. Chem. Sci. 2018, 9, 6325-6339.

(3) Feist, J.; Galego, J.; Garcia-Vidal, F. J. Polaritonic Chemistry with Organic Molecules. ACS Photonics 2018, 5, 205-216.

(4) Herrera, F.; Owrutsky, J. Molecular polaritons for controlling chemistry with quantum optics. $J$. Chem. Phys. 2020, 152, 100902.

(5) Hirai, K.; Hutchison, J. A.; Uji-i, H. Recent Progress in Vibropolaritonic Chemistry. ChemPlusChem 2020, 85, 1981-1988.

(6) Thomas, A.; George, J.; Shalabney, A.; Dryzhakov, M.; Varma, S. J.; Moran, J.; Chervy, T.; Zhong, X.; Devaux, E.; Genet, C.; Hutchison, J. A.; Ebbesen, T. W. Ground-State Chemical Reactivity under Vibrational Coupling to the Vacuum Electromagnetic Field. Angew. Chem. 2016, 128, 11634-11638.

(7) Thomas, A.; Lethuillier-Karl, L.; Nagarajan, K.; Vergauwe, R. M. A.; George, J.; Chervy, T.; Shalabney, A.; Devaux, E.; Genet, C.; Moran, J.; Ebbesen, T. W. Tilting a ground-state reactivity landscape by vibrational strong coupling. Science 2019, 363, 615-619.

(8) Thomas, A.; Jayachandran, A.; LethuillierKarl, L.; Vergauwe, R. M.; Nagarajan, K.; Devaux, E.; Genet, C.; Moran, J.; Ebbesen, T. W. Ground state chemistry under vibrational strong coupling: dependence of thermodynamic parameters on the Rabi splitting energy. Nanophotonics 2020, 9, 249-255.

(9) Vergauwe, R. M. A.; Thomas, A.; Nagarajan, K.; Shalabney, A.; George, J.; Chervy, T.; Seidel, M.; Devaux, E.; Torbeev, V.; Ebbesen, T. W. Modification of Enzyme Activity by Vibrational Strong Coupling of Water. Angew. Chem. Int. Ed. 2019, $58,15324-15328$.

(10) Hirai, K.; Takeda, R.; Hutchison, J. A.; Uji-i, H. Modulation of Prins Cyclization by Vibrational Strong Coupling. Angew. Chem. Int. Ed. 2020, 59, 5332-5335.

(11) Lather, J.; Bhatt, P.; Thomas, A.; Ebbesen, T. W.; George, J. Cavity Catalysis by Cooperative Vibrational Strong Coupling of Reactant and Solvent Molecules. Angew. Chem. Int. Ed. 2019, 58, 10635-10638.

(12) Hiura, H.; Shalabney, A.; George, J. Cavity Catalysis-Accelerating Reactions under Vibrational Strong Coupling. ChemRxiv 2019,

(13) Li, X.; Mandal, A.; Huo, P. Cavity frequencydependent theory for vibrational polariton chemistry. Nature Commun. 2021, 12, 1315.

(14) Grote, R. F.; Hynes, J. T. The stable states picture of chemical reactions. II. Rate constants for condensed and gas phase reaction models. J. Chem. Phys. 1980, 73, 2715-2732.

(15) Ciccotti, G.; Ferrario, M.; Hynes, J. T.; Kapral, R. Dynamics of ion pair interconversion in a polar solvent. J. Chem. Phys. 1990, 93, 7137-7147.

(16) Li, T. E.; Chen, H.-T.; Nitzan, A.; Subotnik, J. E. Quasiclassical modeling of cavity quantum electrodynamics. Phys. Rev. A 2020, 101, 033831.

(17) Flick, J.; Ruggenthaler, M.; Appel, H.; Rubio, A. Atoms and Molecules in Cavities, from Weak to Strong Coupling in Quantum-Electrodynamics (QED) Chemistry. Proc. Natl. Acad. Sci. U. S. A. 2017, 114, 3026.

(18) Vendrell, O. Coherent dynamics in cavity femtochemistry: Application of the multi-configuration time-dependent Hartree method. Chem. Phys. 2018, 509, 55-65. 
(19) Schäfer, C.; Ruggenthaler, M.; Rubio, A. Ab initio nonrelativistic quantum electrodynamics: Bridging quantum chemistry and quantum optics from weak to strong coupling. Phys. Rev. A 2018, 98, 043801 .

(20) Mandal, A.; Krauss, T. D.; Huo, P. PolaritonMediated Electron Transfer via Cavity Quantum Electrodynamics. J. Phys. Chem. B 2020, 124, 6321-6340.

(21) Shin, S.; Metiu, H. Nonadiabatic Effects on the Charge Transfer Rate Constant: A Numerical Study of a Simple Model System. J. Chem. Phys. 1995, 102, 9285-9295.

(22) Flick, L. J.; Appel, H.; Ruggenthaler, M.; Rubio, A. Cavity Born-Oppenheimer Approximation for Correlated Electron-Nuclear-Photon Systems. J. Chem. Theory Comput. 2017, 13, 1616-1625.

(23) Galego, J.; Climent, C.; Garcia-Vidal, F. J.; Feist, J. Cavity Casimir-Polder Forces and Their Effects in Ground-State Chemical Reactivity. Phys. Rev. X 2019, 9, 021057.

(24) Li, T. E.; Nitzan, A.; Subotnik, J. E. On the origin of ground-state vacuum-field catalysis: Equilibrium consideration. J. Chem. Phys. 2020, 152, 234107.

(25) Campos-Gonzalez-Angulo, J. A.; Yuen-Zhou, J. Polaritonic normal modes in transition state theory. J. Chem. Phys. 2020, 152, 161101.

(26) Zhdanov, V. P. Vacuum field in a cavity, lightmediated vibrational coupling, and chemical reactivity. Chem. Phys. 2020, 535, 110767.

(27) Frenkel, D.; Smit, B. Understanding Molecular Simulation; Elsevier: San Diego, 2002.

(28) Miller, W. H.; Schwartz, S. D.; Tromp, J. W. Quantum Mechanical Rate Constants for Bimolecular Reactions. J. Chem. Phys. 1983, 79, 48894898 .

(29) Chandler, D.; Wu, D. Introduction to Modern Statistical Mechanics; Oxford Univ. Press: Oxford, 1987.

(30) Pollak, E. Theory of activated rate processes: A new derivation of Kramers' expression. J. Chem. Phys. 1986, 85, 865-867.

(31) Hänggi, P.; Talkner, P.; Borkovec, M. Reactionrate theory: fifty years after Kramers. Rev. Mod. Phys. 1990, 62, 251-341.

(32) Eyring, H. The Activated Complex in Chemical Reactions. J. Chem. Phys. 1935, 3, 107-115.
(33) Slater, N. B. New Formulation of Gaseous Unimolecular Dissociation Rates. J. Chem. Phys. 1956, 24, 1256-1257.

(34) Chikkaraddy, R.; de Nijs, B.; Benz, F.; Barrow, S. J.; Scherman, O. A.; Rosta, E.; Demetriadou, A.; Fox, P.; Hess, O.; Baumberg, J. J. Single-Molecule Strong Coupling at Room Temperature in Plasmonic Nanocavities. Nature 2016, 535, 127-130. 